\title{
Study on Comparison of Four Different Coatings onto Crystallizer FU Wei ${ }^{1,2, a}$, YONG Yaowei ${ }^{3,4, b}$, DENG Qilin ${ }^{3, c^{*}}$,FANG Hongyuan ${ }^{1, d}$
}

1. State Key Laboratory of Advanced Welding and Joining, Harbin Institute of Technology, Harbin, 150001, PR China

\section{BAOSTEEL Industry Technological Service Co. Ltd, Shanghai, 201900, PR China}

3. School of Mechanical Engineering, Shanghai Jiao Tong University , Shanghai, 200240, PR China

4. School of Mechanical Engineering, Ningxia University, Yinchuan Ningxia, 750021, PR China

a.fw9106@163.com b.yywnxu@163.com c.dengqilin@sjtu.edu.cn d. hyfang@hit.edu.cn

*Corresponding Author: Dengqilin@sjtu.edu.cn

\section{Keywords: Crystallizer; Laser cladding; Electroplating; Plasma spraying}

\begin{abstract}
The $\mathrm{Cr}-\mathrm{Zr}-\mathrm{Cu}$ crystallizer surface was strengthened by laser cladding and obtained the gradient alloy coating. The coating was compared with the other three coating produced by other technology such as electroplating, plasma spraying and plasma spraying with re-melting. The experiments were conducted according their hardness, softening and microstructures, and the results shown that the coating produced by plasma spraying and plasma spraying with re-melting are prone to cracks, the coating produced by electroplating is easily to soften and with low wear resistance, while the coating made by laser cladding shown the best performances for all experiments.
\end{abstract}

\section{Introduction}

Crystallizer is the core component in the continuous casting, and its performance directly affects the production efficiency and quality of billet [1]. In the process of continuous casting, liquid steel closed to the surface of crystallizer is solidified rapidly and then formed certain thickness and shape of solid shell. Solid shell thus be pulled out from the crystallizer, while the steel in center is still in liquid state, as the time passed, the steel is continue cooling and to get the final steel billet. If the shell thickness is not thick enough, the liquid steel will leak at the beginning process, moreover, server accidents could be caused as a result [2], so the initial thickness of shell is extremely important. In order to obtain a certain thickness of the shell, cooling speed should be large enough, which means mold copper plate must possess good thermal conductivity to transfer the heat as quickly as possible. Generally, the crystallizer material is preferred chromium zirconium copper, which has high heat conductivity. There are much slots in back of crystallize for cooling by water and obtaining enough thermal gradient. Therefore, the initial thickness is determined by these two aspects.

In the process of continuous casting, sliding friction exists between the crystallizer and billet, the service life of crystallizer is affected due to the lower wear-resisting of copper. And high temperature corrosion in their working conditions will decrease its quality as well due to the inclusions in the billet surface. In addition, the copper contamination also lowers the billet's quality. Thus, the coating technology can solve these problems, which makes the workpiece has the superior combination properties such as high thermal conductivity, corrosion resistance and high temperature corrosion resistance.

Crystallizer copper plate coating is mainly electroplating [3]. Electroplating is not restricted by the shape and can be used to improve the complicated shape parts. The plating has a dense construct, high corrosion resistance, while the coating hardness is not high enough and has a prone to soft after repeated usage. But also, the needle pores in the plating has a great influence on wear resistance of 
coating. This technology has a serious damage to the environment and thus it is eliminated gradually.

Anther method used to strength the surface is plasma spraying. Plasma spraying rapidly melts alloy powder under high energy beam and spraying to the copper plate surface to form a coating. The hardness of coating by plasma spraying is higher than that of electroplating, while the surface is coarser than that of electroplating, and there are presence of large amounts of porosity, the combination between is mechanical bonding rather than metallurgical combination. While there are some defects appeared on the coating by plasma spraying and electroplating such as wear and peel, and as a result, the service life of crystallizer is decreased[4,5].

In order to improve the coating quality, the re-melting is needed for plasma spraying so as to eliminate the pores in coating and hence refine the bonding to metallurgical combination. The re-melting technology is conducted as the melting point of coating is must be lower than copper substrate. Nevertheless, the melting point of copper is relatively lower, so it restricts the selection of materials of coating.

Laser cladding is a technology using high energy laser beam irradiates the up surface and melts the workpiece and powder simultaneously, and the alloyed coating is formed onto the workpiece surface. The metallurgical bonding is always generated between the coating and substrate with lower dilution and high quality. Laser cladding can be used to clad the material with higher melting point than that of substrate and thus it has a wide selection in material of coating $[6,7]$.

\section{Preparation of Samples}

In order to compare the advantages and disadvantages of different coating, there are four samples have been prepared, as listed in Table 1

Table 1 Preparation of Four Samples

\begin{tabular}{|c|c|}
\hline sample & Coating methods \\
\hline 1 & electroplating \\
\hline 2 & Plasma spraying \\
\hline 3 & Plasma spraying with re-melting \\
\hline 4 & Laser cladding $\dagger$ \\
\hline
\end{tabular}

$\dagger$ Prior to experiments, the first layer of nickel has been melted onto the copper substrate to eliminate the thermal expansion effect on the future coating due to the closed thermal expansion coefficient of copper and nickel. Therefore, the cobalt alloy coating was made onto the nickel coating with the hardness of about 45HRC.

\section{Procedure of Experiments and Discussion}

\subsection{Wear Testing}

The crystallizer works under the server wear condition. And to simulate the wear condition in reality, the room temperature wear testing had been carried out to estimate the behaviors of four coating.

The electroplating coating is with lower hardness and to soften in high temperature, so the wear property is far weaker than that of laser cladding [8]. In this study, the ring-disk sliding wear testing was conducted for the other three coating such as laser cladding, plasma spraying and with re-melting. The wear couple is $316 \mathrm{~L}$, the weight loss of $316 \mathrm{~L}$ and coating were measured respectively per hour. And the wear loss compared with the $316 \mathrm{~L}$ weight loss is shown in Fig. 1 


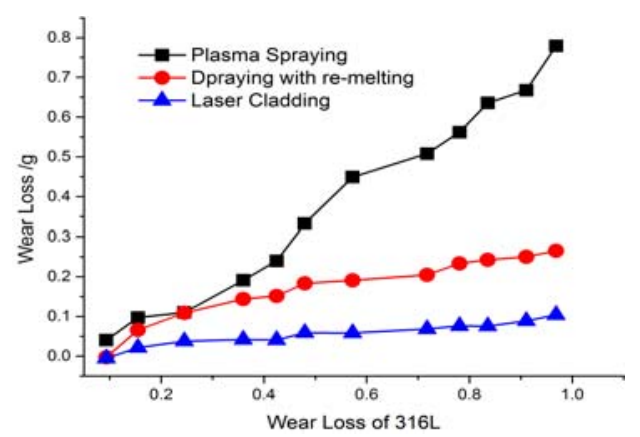

Fig. 1 Comparison of Wear Loss of Three Coating

It can be found from Fig. 1 that the wear resistance of laser cladding is best in three coating. The ratio of $316 \mathrm{~L}$ to plasma spraying, plasma spraying with re-melting and laser cladding is $0.82,0.26$ and 0.11 respectively. Thus the rank of wear resistance from high to low is laser cladding, plasma spraying and re-melting and plasma spraying. Specially, the wear resistance of laser cladding coating is much better than that of the other two coating.

\subsection{Thermal Shock Experiments}

At the beginning of casting, the copper crystallizer will experience the process from room temperature to liquid steel temperature as the liquid steel is poured into the crystallizer. Thus, the copper plate will endure the huge thermal shock. Under the repeated thermal shocks, the crystallizer plate occurs the corrosion, cracking and peeling. In order to mirror this process, the thermal shock experiments had been carried out to investigate its behaviors. The four samples were heated to $950^{\circ} \mathrm{C}$ and lasted for 10 minutes, and then immersed the hot samples into the cool water to inversely simulate the thermal shock process of crystallizer. In the process, if the cracks can be found onto the sample coating surface, the experiment for this failure sample was ended. The samples of thermal shocks were shown in Fig. 2

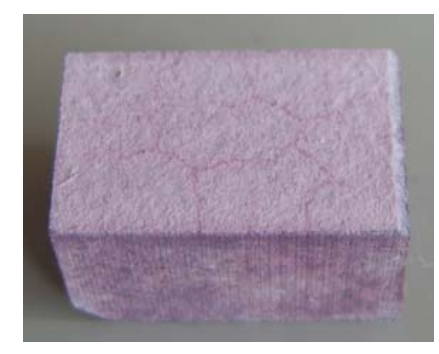

a) Plasma Spraying Coating

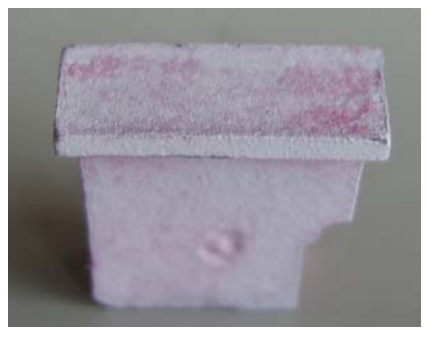

c) Electroplating Coating

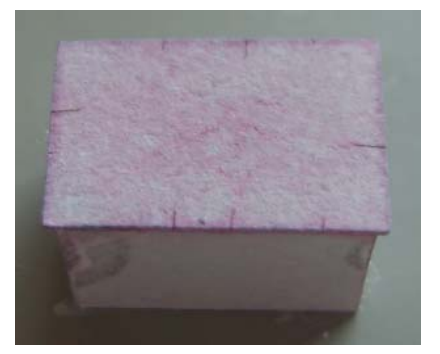

b) Plasma Spraying Coating with Re-melting

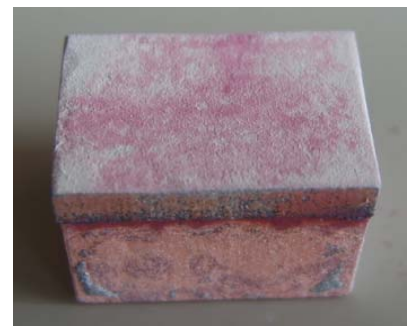

d) Laser Cladding Coating

Fig. 2 Surface Morphology after Thermal Shock

Reveled by Fig. 2, the cracks were firstly shown in sample of plasma spraying coating after many times thermal shocks, as shown in Fig. 2 (a). Then it appeared onto the plasma spraying with re-melting samples coating after continued thermal shocks, as shown in Fig. 2 (b). While there no clear cracks were found onto the other two coatings. Thus, it can be concluded that the electroplated coating and laser cladding coating is with high thermal shock resistance. Taking the wear resistance into account, the laser cladding coating showed the better performance. 


\subsection{Comparison of Micro-Hardness after Thermal Shock}

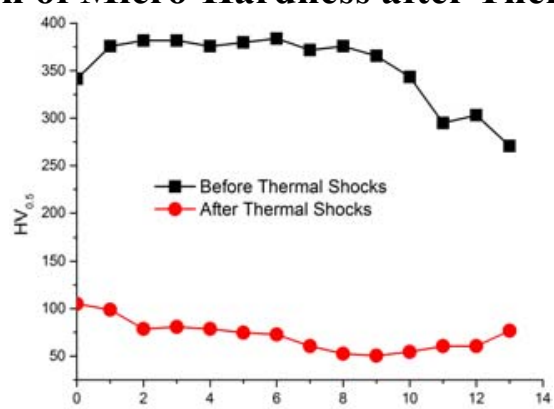

a) Comparison of Hardness of sample 1

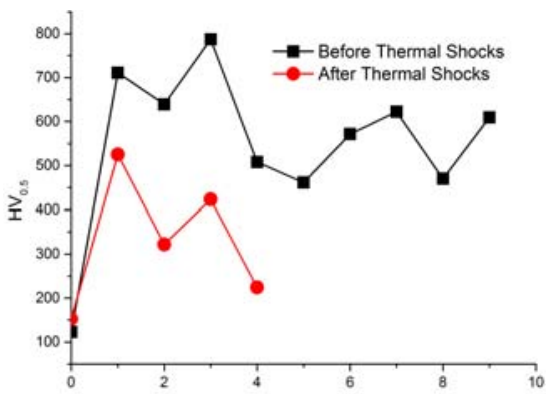

c) Comparison of Hardness of sample 3

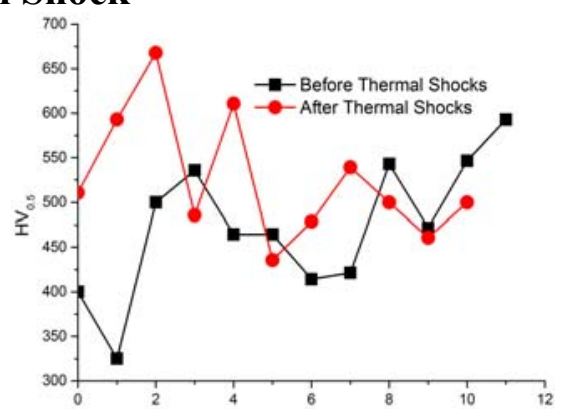

b) Comparison of Hardness of sample 2

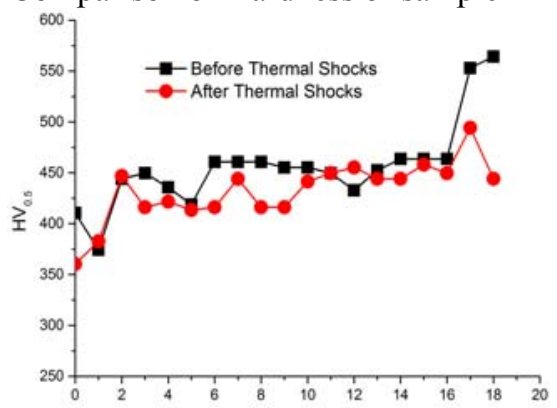

d) Comparison of Hardness of samples 4

Fig. 3 Hardness of Coating before and after Thermal Shocks

The hardness of four coating has been measured before and after thermal shocks, the hardness of laser cladding coating was measured only onto the cobalt layer. The comparison of micro-hardness before and after thermal shocks was shown in Fig. 3 .

It can be seen from Fig. 3 that the laser cladding coating has a stronger resistance to high temperature soften tendency. The average hardness was decreased from $380 \mathrm{HV}_{0.5}$ before thermal shock to $60 \mathrm{HV}_{0.5}$ after thermal shocks. There was server softening phenomena happened near the interface after thermal shock for plasma spraying coating, while the hardness have no clear change in middle and up-surface and the average hardness is about $500 \mathrm{HV}_{0.5}$. Hardness of plasma spraying with re-melting coating decreased from $650 \mathrm{HV}_{0.5}$ to $350 \mathrm{HV}_{0.5}$. While the hardness of coating from laser cladding was about $450 \mathrm{H} \mathrm{V}_{0.5}$, and have no clear change. Because the thermal shocks of plasma spraying coating repeated shortly, the decrease in hardness was not evident, and thus had no valuable meaning. Comparison among those coating, the coating produced by laser cladding was best evidently.

\subsection{Comparison of Micro-structure after Thermal Shock}

The microstructure of four coating had been observed before and after thermal shocks, as shown in Fig. 4.

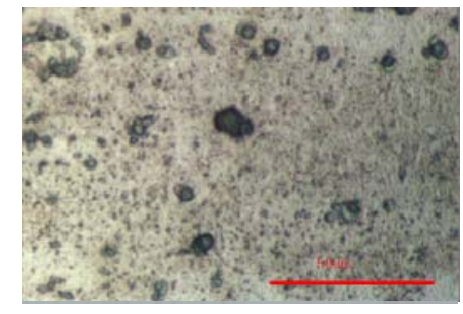

a) Before Thermal Shocks of Sample 1

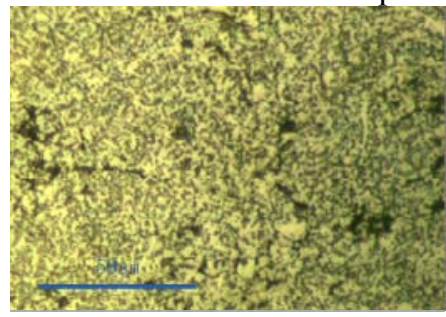

d) After Thermal Shocks of Sample 2

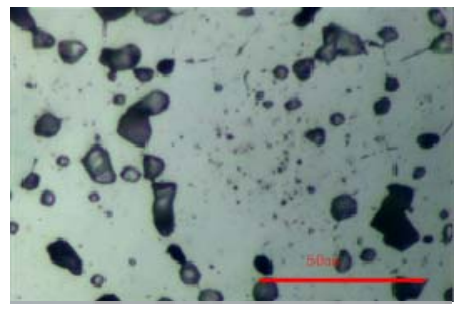

b) After Thermal Shocks of Sample

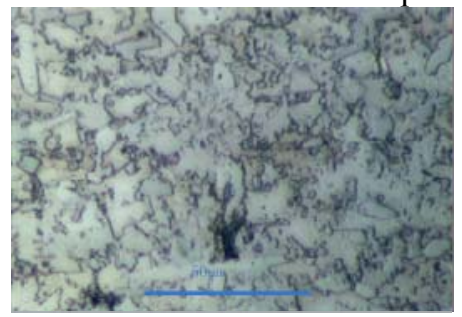

e) Before Thermal Shocks of Sample 3

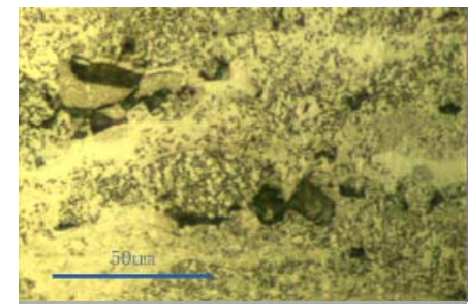

c) Before Thermal Shocks of Sample 2

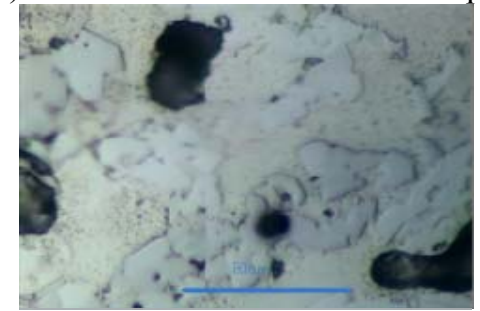

f) After Thermal Shocks of Sample 3 


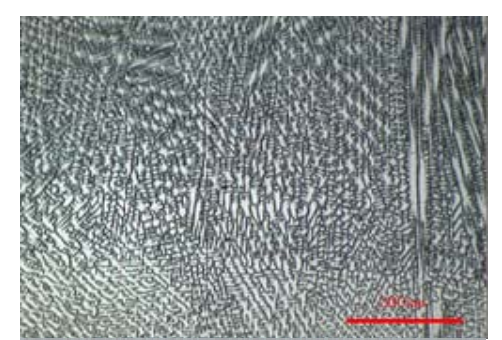

g) Before thermal shocks of sample 4

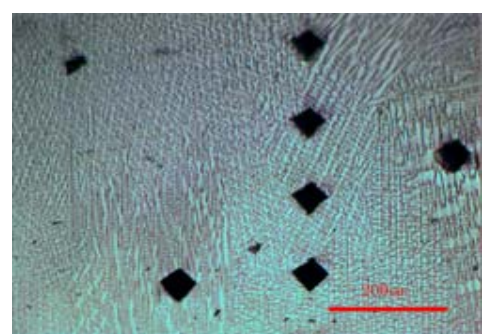

h) After thermal shocks of sample 4

Fig. 4 Micro-structure of Four Coating before and after Thermal Shocks

The coating microstructures before and after thermal shocks of electroplating were shown in Fig. 4(a) and Fig. 4(b). It can be found that there are many hard particles embedded in coating before thermal shocks, which can improve the coating's hardness and wear resistance due to the dispersion strengthening effect. After repeatedly thermal shocks, the hard particles were precipitated from matrix and accumulated, simultaneously; the needle-shaped phase was formed close to the edge of hard particles. This will cause decrease in hardness of coating, as a result, the whole hardness of coating was declined seriously. It also can be inferred that the wear resistance of coating could be lowered; consequently, the service life of crystallizer will be deteriorated as well.

The coating microstructures before and after thermal shocks of plasma spraying were shown in Fig. 4(c) and Fig. 4(d). The figures revealed that there are many pores in sparse coating, and the structure distributed non-uniformly before thermal shocks became uniformity after thermal shocks and pores were disappeared partially. The high temperature was up to 950 degrees, it is very close to the melting point of coating, thus the repeated thermal shock can eliminate some pores. While the structure was still coarse, and pores were still in coating, these all had inverse affects on the coating characteristics.

The coating microstructures before and after thermal shocks of plasma spraying with re-melting were shown in Fig. 4(e) and Fig. 4(f). The figures revealed that there two phases were recognized clearly. Before thermal shocks, the boundary of two phases is tortuous and distribution is evenly tiny. After repeated thermal shocks, a phase was precipitated and accumulated, and the boundary of phase became straight and regular. The hardness of coating before thermal shocks is $670 \mathrm{HV}_{0.5}$, and it is $530 \mathrm{HV}_{0.5}$ for substrate. After thermal shocks, the precipitated particles held the hardness of 670 $\mathrm{HV}_{0.5}$, while it is decreased to $400 \mathrm{HV}_{0.5}$ for substrate. This due to the precipitated hard particles has the strengthening effect on the hardness. It also can be found from Fig. 4(f) that the pores were accumulated and enlarged.

The coating microstructures before and after thermal shocks of laser cladding were shown in Fig. 4(e) and Fig. 4(f). There were dendrite crystal structure distributed uniformly in coating, and the thermal shocks had little influence on the microstructure. And the harnesses were all constant of 450 $\mathrm{HV}_{0.5}$.

Compared the microstructure of all four coating, it can be concluded that the coating made by laser cladding has unique advantages for thermal shock resistance.

\section{Conclusion}

The coatings produced onto the copper crystallizer by electroplating, plasma spraying, plasma spraying with re-melting and laser cladding were compared and studied, and the results were drawn by the studies as follow:

1) For wear sliding test, the coating produced by laser cladding is better than that of the other three coatings;

2) For thermal shocks testing, the coating made by plasma spraying shows worst performance in crack resistance, and followed by plasma spraying and plasma spraying with re-melting, while the coating produced by electroplating and laser cladding shows the best performance in crack resistance;

3) From the observation of microstructure, the coating by electroplating is softening severely, coating made by plasma spraying with re-melting is slightly better than that of former, and there is 
no clear softening in laser cladded coating;

4) The coating made by laser cladding and electroplating show the uniformly dense structure before thermal shocks, while there are many pores in coating made by electroplating after thermal shocks, but the coating shows the better identity structure with the structure before thermal shocks.

\section{Acknowledgment}

The authors gratefully thank the financial support from National Natural science foundation of China (No 51275303)

\section{Reference}

[1] Wang Yazhen,Ye Jin,Zahang Yan. "Process and equipmeng of Continuous casting”.Metallurgical Industry Press,Beijing, 2007.

[2] Fang Keming,Yu Yanming,Xue Yuezhong etc,'Discusion about designing and mainatining of the copper plate of CCM mold", Heavy machinery, 2009, (3):1-6.

[3] Liu Fang, Liu Changsheng, Tao Xingqi etc. "Progress in surface treatment of copper crystallier",Surface Technology, 2006, 35(3):1-3

[4] Ma Guangqun, "Clad layer exfoliation reason of copper plate for clab continuous caster crystallizer and improvement", Gansu Metalllurgy, 2012, 34(2):1-4

[5] Zhang Hongjie,Wen Maoyuan,Hou Zhen etc. "Analysis on failure of mold copper plate of continuous caster and counter measures", Steelmaking ,2012, 28(1):70-73,7.

[6] Chen S, Liang J, Liu C, etc. "Preparation of a novel Ni/Co-based alloy gradient coating on surface of the crystallizer copper alloy by laser". Applied Surface Science, 2011, 258(4): 1443-1450.

[7]. Yan $\mathrm{H}$, Zhang $\mathrm{P}, \mathrm{Yu} \mathrm{Z}$, etc. "Development and characterization of laser surface cladding $(\mathrm{Ti}, \mathrm{W}) \mathrm{C}$ reinforced $\mathrm{Ni30Cu}$ alloy composite coating on copper". Optics and Laser Technology, 2012, 44(5): 1351-1358.

[8]. Gao Sen, Jia Zhiguo,Deng Qilin. "Researches on Laser Cladding Strengthening Technology on Copper Crystallizer Surface and Comparison with Electroplating Technology". Electromachining \& Mould, 2013, (01): 31-36. 